\title{
Transcutaneous electrical diaphragmatic stimulation reduces the duration of invasive mechanical ventilation in patients with cervical spinal cord injury: retrospective case series
}

\author{
Gregory Lui Duarte $\mathbb{1}^{1,2} \cdot$ Aldrin Lucas Bethiol ${ }^{3,4} \cdot$ Ligia dos Santos Roceto Ratti $^{5} \cdot$ Gabriel Franco $^{6} \cdot$ Rui Moreno ${ }^{7}$. \\ Rodrigo Marques Tonella ${ }^{8}$. Antonio Luis Eiras Falcão ${ }^{9}$
}

Received: 18 October 2019 / Revised: 19 March 2021 / Accepted: 26 March 2021

(c) The Author(s), under exclusive licence to International Spinal Cord Society 2021

\begin{abstract}
Study design Retrospective case series.

Objectives To compare individuals with cervical spinal cord injury (SCI) submitted to transcutaneous electrical diaphragmatic stimulation (TEDS) or a standard weaning protocol (SWP) according to the following variables: invasive mechanical ventilation (IMV) time, ventilator weaning time, intensive care unit (ICU) length of stay, and overall hospital length of stay. Settings Tertiary university hospital. Clinical Hospital of Campinas State University_UNICAMP_Campinas (SP), Brazil. Methods Retrospective case study investigating ICU patients submitted to tracheostomy due to cervical SCI at a tertiary university hospital (Clinical Hospital of Campinas State University, Brazil). Data were extracted from medical records of patients seen between January 2007 and December 2016. According to medical records, four patients were submitted to TEDS and six to a SWP. Provision of training to patients in the TEDS group was based on consensus medical decision, preference of the physical therapy team and availability of electrostimulation equipment in the ICU.

Results Total IMV time in the TEDS and the SWP group was $33 \pm 15$ and $60 \pm 22$ days, respectively. Length of stay in ICU in the TEDS and the SWP group was $31 \pm 18$ and $63 \pm 45$ days, respectively.

Conclusion TEDS appears to influence the duration of IMV as well as the length of stay in ICU. This physiotherapeutic intervention may be a potentially promising tool for treatment of patients with SCI. However, randomized clinical trials are warranted to support this assumption.
\end{abstract}

\section{Introduction}

Individuals with spinal cord injury (SCI) may develop severe respiratory complications, such as decreased vital capacity and loss of abdominal, intercostal and diaphragm muscle strength, which may lead to reduced peak expiratory flow, increased effort of breathing, and tracheobronchial secretion buildup [1]. Cervical spinal cord injuries (CSCI), especially those located between $\mathrm{C} 3$ and $\mathrm{C} 5$, may damage phrenic nerve roots and lead to respiratory failure. Persons with acute $\bowtie$ Gregory Lui Duarte

gregoryluiduarte@hotmail.com

1 Intensive Care Unit, Campinas State University-UNICAMP, Campinas, SP, Brazil

2 Department of Surgery, Campinas State University-UNICAMP, Campinas, SP, Brazil

3 Hospital Sancta Maggiore de Pinheiros, São Paulo, SP, Brazil

4 Hospital do Coração, São Paulo, SP, Brazil

5 Clinical Hospital of Campinas State University, Campinas, SP, Brazil
6 Department of Statistics, Campinas State University-UNICAMP, Campinas, SP, Brazil

7 Neurocritical and Trauma Intensive Care Unit, Hospital de São José, Centro Hospitalar Universitário de Lisboa Central, Nova Medical School, Lisbon, Portugal

8 Department of Physiotherapy, School of Physical Education, Physiotherapy and Occupational Therapy-EEFFTO-Federal University of Minas Gerais-UFMG, Belo Horizonte, Minas Gerais, Brazil

9 Department of Surgery, School of Medical Sciences, Campinas State University-UNICAMP, Campinas, SP, Brazil 
CSCI often require invasive mechanical ventilation (IMV) followed by prolonged weaning, which ultimately requires inspiratory muscle training and rehabilitation [2].

Cardiorespiratory complications such as atelectasis, pneumonia, sepsis, bradycardia, postural hypotension, and respiratory failure are the leading causes of death in hospitalized patients with SCI [3]. Patients with acute SCI requiring prolonged IMV may develop disuse atrophy of the diaphragm [4].

Intramuscular implants and phrenic nerve stimulation can be used to assist with rehabilitation of ventilator-dependent persons. These therapeutic interventions provide some level of independence from mechanical ventilation, improve speech, and reduce the risk of respiratory tract infections, with significant improvement in patient quality of life [5]. However, the initial cost of these interventions is high, the implant surgery has inherent risks, and management of nerve stimulators requires specific training [5].

Neuromuscular electrical stimulation (NMES) is commonly used in physical therapy to increase muscle strength and promote muscle hypertrophy. In NMES, an electric current is used to depolarize the membrane of alpha-motor neurons, triggering an action potential that generates muscle contraction [6-8]. NMES applied to respiratory rehabilitation is called transcutaneous electrical diaphragmatic stimulation-TEDS [9]. This physiotherapeutic intervention is inexpensive and virtually risk free. However, its effects on critically ill patients have seldom been reported and treatment protocols and guidelines remain to be established [9].

In a systematic review addressing the use of respiratory muscle training in tetraplegic patients, Tamplin and Berlowitz [10] emphasized the need of further studies to determine the ideal intensity of respiratory muscle training, the duration of training-related muscle strength and resistance gains, and the long-term benefits derived from this type of training.

This study set out to compare individuals with CSCI submitted to TEDS or to a standard weaning protocol (SWP). The following variables were examined: duration of IMV, ventilator weaning time (WT), intensive care unit (ICU) length of stay, and overall hospital length of stay.

\section{Methods}

This is a retrospective case study investigating ICU patients submitted to tracheostomy (TOT) due to cervical SCI at a tertiary university hospital (Clinical Hospital of Campinas State University, Brazil). Data were extracted from medical records of patients seen between January 2007 and December 2016. Inclusion criteria were as follows: CSCI with tetraplegia, AIS (American Spinal Injury Association Impairment Scale) grade A injury upon admission [11]; intact phrenic nerve function on clinical assessment [11]; IMV followed by prolonged weaning; TOT; complete medical record; computed tomographic assessment with corresponding report. Patients who failed to meet these criteria were excluded.

According to medical records, four patients were submitted to TEDS and six to a SWP. Provision of training to patients in the TEDS group was based on consensus medical decision, preference of the physical therapy team, and availability of electrostimulation equipment in the ICU, as described in medical records.

The SWP consisted of intermittent oxygen-driven nebulization delivered via TOT mask for progressively longer periods of time until successful disconnection from IMV was achieved (i.e., ventilator-free breathing for $48 \mathrm{~h}$ ). IMV was resumed for $12 \mathrm{~h}$ in order to provide respiratory muscle rest in the following cases: respiratory rate higher than 35 breaths/min, heart rate higher than 140 beats/min, systolic blood pressure higher than 180 or lower than $90 \mathrm{mmHg}$, peripheral oxygen saturation under $90 \%$, regardless of restlessness, sweating, altered levels of consciousness, or thoracoabdominal asynchrony [12].

In patients in the TEDS group, TEDS was combined with the SWP. Training consisted of two daily 20-min sessions 7 days a week. Electrical stimulation was triggered manually once every two breaths using verbal cues and a Phrenics Dualpex Quark (Piracicaba, Brazil) device with the following settings: frequency of 30 hertz, pulse width of $1 \mathrm{~ms}$, rise time of $0.7 \mathrm{~ms}$, and current intensity of 60 milliamps. A dual channel unit with self-adhesive electrodes $\left(3 \mathrm{M}^{\circledR}\right.$, Saint Paul, MN, USA) was used. Electrodes were attached to the left and right midaxillary line at the level of the sixth, seventh, and eighth intercostal spaces, and to the paraxiphoid region [13]. Inspiratory training was discontinued after patients were successfully disconnected from IMV.

The following variables were analyzed using $\mathrm{R}$ version 3.6.2: time of IMV via orotracheal tube, time of IMV via TOT, ventilator WT, total IMV time, ICU length of stay, overall hospital length of stay, Sepsis-related Organ Failure Assessment (SOFA), and Acute Physiology and Chronic Health Evaluation (APACHE II) scores. Numerical variables were expressed as means and standard deviations. Categorical variables were described as relative frequencies. Mean values of time-invariable variables were compared using the $t$-test. The level of significance was set at $5 \%(p<$ $0.05)$. Given the small number of observations, statistical analyses were based primarily on descriptive statistics and graphical representations such as box and scatter plots.

\section{Results}

In total, 89 patients were admitted to the ICU due to SCI from January 2007 to December 2016. Of these, 30 were diagnosed with CSCI and 13 underwent TOT. Tracheostomized patients 
Fig. 1 Study flowchart. Study flowchart of patients' sample.

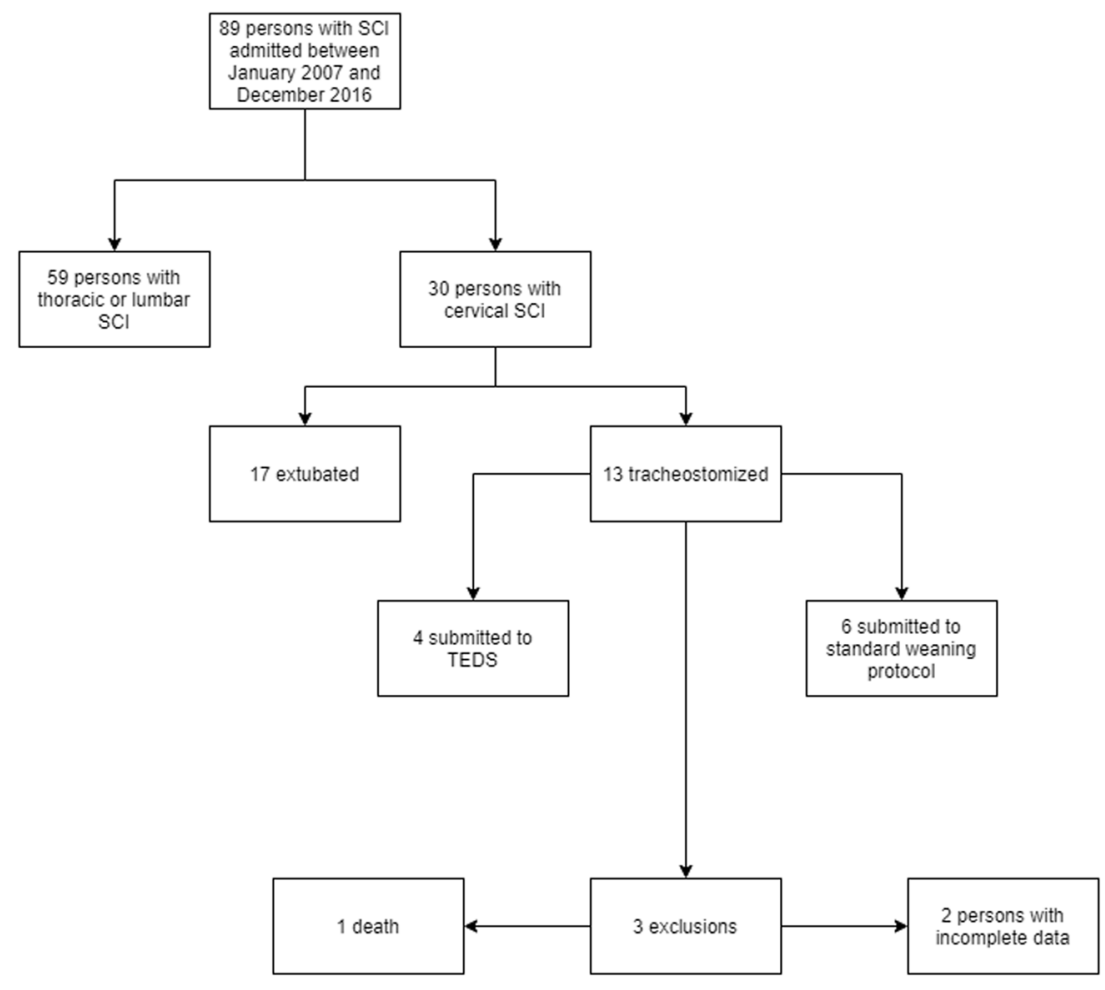

were allocated to the TEDS (four) or the SWP (six) group. One patient was excluded due to inability to complete the ventilator weaning protocol and progression to death and two due to incomplete records (Fig. 1).

Demographic data of selected patients and variables of interest are shown in Table 1. Total IMV time in the TEDS and the SWP group was $33 \pm 15$ and $60 \pm 22$ days, respectively. Length of stay in ICU in the TEDS and the SWP group was $31 \pm 18$ and $63 \pm 45$ days, respectively. Causes of SCI in this sample are listed in Table 2. Car accidents were the major cause of SCI, accounting for $40 \%$ of cases.

IMV duration in patients in the TEDS and the SWP group is shown in Fig. 2. Time on IMV was 1.77 times shorter in patients in the TEDS relative to patients in the SWP group.

Length of stay in ICU of patients in the TEDS and the SWP group is shown in Fig. 3. Mean ICU length of stay was 2.54 times shorter in patients in the TEDS group relative to patients in the SWP group.

Training duration (TEDS group) and the time elapsed from start of training to successful ventilator withdrawal are shown in Table 3. The mean number of training sessions required for ventilator withdrawal was 47, spread across 23 days on average.

\section{Discussion}

Spinal cord injuries affect primarily young adults and therefore have significant social and economic impacts [14].
Table 1 Demographic data and variables of interest.

\begin{tabular}{lllll}
\hline & SWP $^{\mathrm{a}}$ & TEDS $^{\mathrm{a}}$ & Diff $^{\mathrm{a}}$ & $p$ value \\
\hline$N$ & 6 & 4 & & - \\
Sex & & & & \\
$\quad$ Male & $83 \%$ & $75 \%$ & & - \\
$\quad$ Female & $16 \%$ & $25 \%$ & & - \\
Age (years) & $29 \pm 5$ & $28 \pm 14$ & & 0.97 \\
APACHE II score & $8 \pm 3.1$ & $13 \pm 7.2$ & & 0.22 \\
SOFA score & $4.1 \pm 2.2$ & $6.5 \pm 3.4$ & & 0.28 \\
IMV (days) & & & & \\
$\quad$ OTT & $7.8 \pm 7.9$ & $3.5 \pm 1.9$ & $-4.3 \pm 6.3$ & - \\
$\quad$ TOT & $52 \pm 19$ & $30 \pm 15$ & $-21 \pm 17$ & - \\
$\quad$ IMV TOTAL & $60 \pm 22$ & $33 \pm 15$ & $-26 \pm 19$ & - \\
Weaning time (days) & $50 \pm 19$ & $28 \pm 15$ & $-21 \pm 17$ & - \\
Hospital stay (days) & & & & - \\
$\quad$ Overall stay & $81 \pm 44$ & $60 \pm 32$ & $-21 \pm 40$ & - \\
$\quad$ ICU stay & $63 \pm 45$ & $31 \pm 18$ & $-32 \pm 37$ & - \\
\hline
\end{tabular}

SWP standard, weaning protocol, TEDS transcutaneous electric diaphragmatic stimulation, Diff arithmetic difference, APACHE II Acute Physiology and Chronic Health Evaluation, SOFA Sepsisrelated Organ Failure Assessment, IMV invasive mechanical ventilation, $O T T$ time of invasive mechanical ventilation time via orotracheal tube (days), TOT time of invasive mechanical ventilation time via tracheostomy (days), IMV TOTAL total time spent on invasive mechanical ventilation, Weaning time time elapsed between first spontaneous breathing trial and successful ventilator withdrawal, Overall stay time from hospital admission to discharge, ICU stay time from ICU admission to discharge to regular hospital ward.

${ }^{\mathrm{a}}$ Mean \pm standard deviation. 
Table 2 Causes of spinal cord injury.

\begin{tabular}{llllllll}
\hline Patient & Admission year & Group & Sex & Age & Cause of injury & Injury level & AIS grade \\
\hline 1 & 2016 & TEDS & M & 17 & Diving & C5/6 & A \\
2 & 2015 & TEDS & M & 50 & Fall & C5/6 & A \\
3 & 2016 & TEDS & M & 24 & Car accident & C4/6 & A \\
4 & 2014 & TEDS & F & 24 & Car accident & C4/5 & A \\
5 & 2011 & SWP & M & 22 & Car accident & C2/4 & A \\
6 & 2014 & SWP & M & 29 & Unknown & C5/6 & A \\
7 & & & & & & T3/6 & A \\
8 & 2015 & SWP & M & 29 & Diving & C4/5 & A \\
9 & 2012 & SWP & F & 25 & Car accident & C4/5 & A \\
10 & 2015 & SWP & M & 34 & Diving & C6/7 & A \\
\hline
\end{tabular}

AIS American Spinal Injuries Association Impairment Scale (AIS score refers to function and sensation below injury level: A-no motor or sensory function; B-sensory function but no motor function; Csensory function with severe motor function compromise).

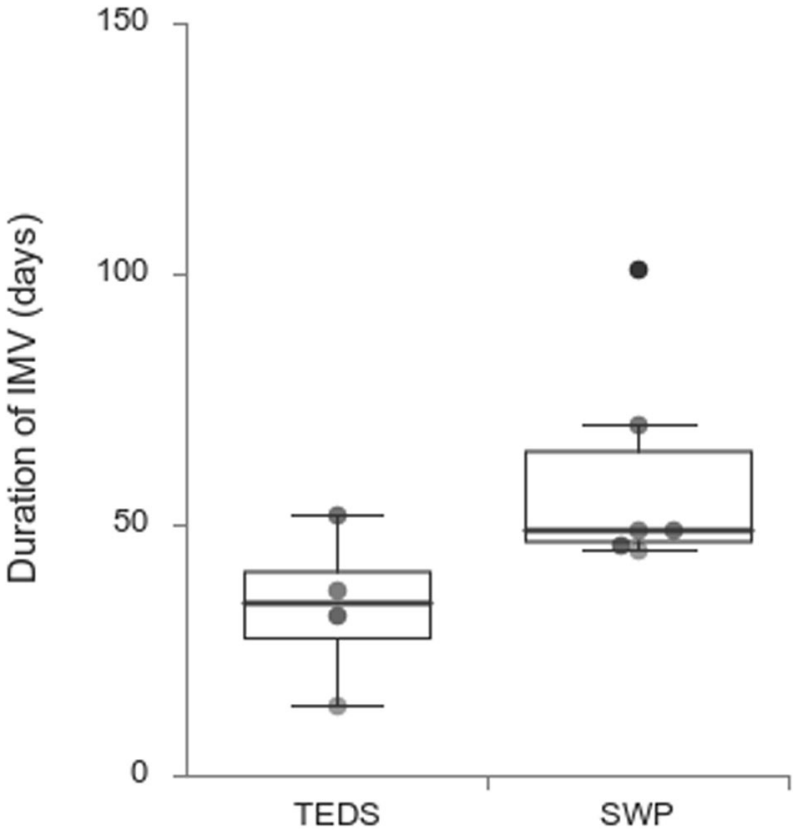

Fig. 2 The ordinate indicates the duration of invasive mechanical ventilation (IMV) in days. SWP standard weaning protocol, TEDS transcutaneous electrical diaphragmatic stimulation.

In a Brazilian cohort study conducted with 348 patients with SCI, Belluci et al. [15] identified an epidemiologic trend with regard to patient age (mean age, $35 \pm 15$ years). Patients in this sample were mostly younger $(28 \pm 14$ and $29 \pm 5$ years of age; TEDS and the SWP group, respectively).

The incidence of SCI in men and women varies, but studies overall report a ratio of at least 2:1 [16]. Likewise, this sample comprised primarily males (80\%). Similar findings have been reported by Chen et al. [17].

Injury severity did not differ significantly between groups according to SOFA and APACHE II scores $(p=0.28$ and $p=0.22$, TEDS and SWP group,

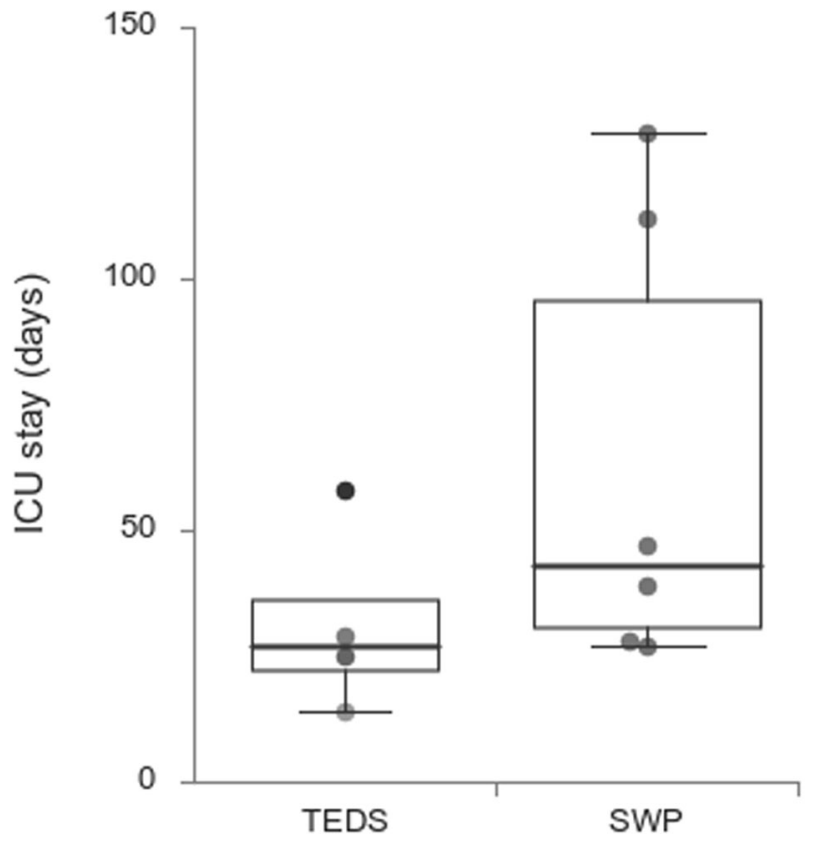

Fig. 3 The ordinate indicates the duration of length of stay in intensive care unit (ICU) in days. SWP standard weaning protocol, TEDS transcutaneous electrical diaphragmatic stimulation.

respectively). Cases in this study (mean SOFA scores, $6.5 \pm 3.4$ and $4.1 \pm 2.2$, TEDS and SWP group, respectively) were less severe than those reported by Stein et al. [18] (mean SOFA score, $8.8 \pm 2.6$ ), despite the lack of significant intergroup differences. Stein et al. [18] suggested the higher the AIS grade, the higher the changes of organ failure, particularly respiratory failure. Accordingly, patients in this sample were diagnosed with AIS grade A injuries and progressed to respiratory failure requiring IMV followed by prolonged weaning.

Levine et al. [4] observed that controlled IMV for as little as $18 \mathrm{~h}$ was enough to induce diaphragm atrophy in healthy subjects. Goligher et al. [19] described multifactorial 
Table 3 Characteristics of training sessions.

\begin{tabular}{lll}
\hline Training sessions & & \\
\hline Patient & Number of sessions & Number of days \\
\hline 1 & 20 & 10 \\
2 & 60 & 30 \\
3 & 44 & 22 \\
4 & 66 & 33 \\
Mean \pm SD & $47 \pm 20$ & $23 \pm 10$ \\
\hline
\end{tabular}

Training sessions of patients in the TEDS group; number of sessions required to achieve successful IMV withdrawal; number of days between the first training session and successful ventilator withdrawal. $S D$ standard deviation.

reduction in diaphragm thickness suggestive of atrophy in patients undergoing prolonged IMV. In this study, patients in the SWP group required $60 \pm 22$ days of IMV, whereas patients in the TEDS group required only $33 \pm 15$ days. Following unsuccessful attempts to withdraw IMV, patients in this sample were thought to require specific inspiratory muscle training and TEDS was the technique of choice.

Transcutaneous electric diaphragmatic stimulation refers to the application of an electric current to elicit muscle contraction and can therefore be used in inspiratory muscle training. Results obtained are intensity, frequency, and interval dependent. In this study, inspiratory muscles were trained twice daily 7 days a week (47 sessions per patient on average). Patients submitted to TEDS spent less time in ICU. However, length of stay in hospital was not affected. This paradoxical finding may reflect discontinuation of muscle training immediately after discharge from ICU. In a trial conducted by Postma et al. [20], 19 patients with SCI were submitted to inspiratory muscle training using a Threshold IMT inspiratory muscle trainer (Threshold IMT device HS730, Respironics, NJ, USA). The training protocol in that study consisted of five weekly training sessions for 8 weeks (39 sessions on average). Short-term gains in inspiratory muscle strength were lost after the protocol was discontinued, suggesting continued training is vital to maintain inspiratory muscle strength.

In this study, patients in both groups were successfully weaned from IMV. However, in all cases the need of IMV was determined by the CSCI per se, which affected intercostal and accessory muscle function, leading to diaphragm overload, reduced tidal volume, decreased pulmonary compliance, and loss of abdominal support during the respiratory cycle [21]. Septic and respiratory failure episodes associated with asynchrony and intermittent respiratory support suggest these patients also sustained diaphragmatic myotrauma.

Myotrauma may result from several mechanisms, including inappropriate management of mechanical ventilation (over- or under-assistance) and eccentric diaphragmatic contractions, especially during asynchrony episodes induced by impaired cough reflex, a common occurrence following SCI. According to Goligher et al. [19], these factors may account for repeated episodes of myotrauma, which may lead to diaphragmatic inflammation and ultimately to sarcopenia and atrophy. Related episodes of sepsis and malnutrition also contribute to IMV dependence. Therefore, muscle training may play an important role in the recovery of these patients.

In this study, patients submitted to TEDS spent considerably less time on IMV and in the ICU than patients in the SWP group (1.77 and 2.54 times less, respectively). Likewise, in a systematic review of nine studies addressing respiratory muscle training, Vorona et al. [22] observed a similar reduction in IMV duration in patients submitted to this type of training.

Findings reported in the literature suggest that inspiratory muscle training is a simple, low-cost intervention conducive to maximal inspiratory pressure (MIP) increase [23]. In spite of considerably shorter IMV duration in patients submitted to TEDS in this study, inspiratory muscle training had no significant impacts on their WT. In a randomized trial conducted by Condessa et al. [24], use of a Threshold IMT inspiratory muscle trainer (Threshold IMT device HS730 Respironics, NJ, USA) twice daily 7 days a week also led to an increase in MIP with no impact on patient WT.

Findings of this study suggest that TEDS is a potentially promising inspiratory muscle training tool after prolonged weaning. Still, study limitations must be acknowledged. First, retrospective design limited study conclusions. Second, small sample size compromised the robustness of statistical analyzes. Inability to quantify training load and lack of similar electrical stimulation trials precluded a broader discussion and prevented assertive conclusions.

\section{Conclusion}

This is a retrospective study with a small sample size. Still, findings suggest that TEDS influences the duration of IMV as well as the length of stay in ICU, and is a potentially promising tool for treatment of patients with SCI. However, randomized clinical trials are warranted to support this assumption.

\section{Data availability}

The data sets used and analyzed during the current study are available from the corresponding author on reasonable request.

\section{Compliance with ethical standards}

Conflict of interest The authors declare no competing interests. 
Ethical approval This study was approved by the local Ethics Committee (Process No. CAAE 68040417.7.0000.5404).

Informed consent Waiver of informed consent was obtained due to the retrospective nature of the study. Personal information was protected by electronic encryption; access was limited to data relevant to the study.

Publisher's note Springer Nature remains neutral with regard to jurisdictional claims in published maps and institutional affiliations.

\section{References}

1. Umphred D. Reabilitação neurológica. Brazil: Elsevier Brasil; 2011.

2. Sartori NR, Melo MR. Necessidades no cuidado hospitalar do lesado medular. Medicina. 2002;35:151-9.

3. Claxton AR, Wong DT, Chung F, Fehlings MG. Predictors of hospital mortality and mechanical ventilation in patients with cervical spinal cord injury. Can J Anaesth. 1998;45:144.

4. Levine S, Nguyen T, Taylor N, Friscia ME, Budak MT, Rothenberg $\mathrm{P}$, et al. Rapid disuse atrophy of diaphragm fibers in mechanically ventilated humans. N Engl J Med. 2008;358:1327-35.

5. Jarosz R, Littlepage M, Creasey G, McKenna S. Functional electrical stimulation in spinal cord injury respiratory care. Top Spinal Cord Inj Rehabil. 2012;18:315-21.

6. Ragnarsson KT. Functional electrical stimulation after spinal cord injury: current use, therapeutic effects and future directions. Spinal Cord. 2008;46:255-74.

7. Marsolais EB, Kobetic R. Functional electrical stimulation for walking in paraplegia. JBJS. 1987;69:728-33.

8. Pfurtscheller G, Müller GR, Pfurtscheller J, Gerner HJ, Rupp R. 'Thought'-control of functional electrical stimulation to restore hand grasp in a patient with tetraplegia. Neurosci Lett. 2003;351:33-6.

9. Sarmento GJV. Respiratory physiotherapy in critically ill patients: clinical routines. 2nd rev. ed. São Paulo: Manole; 2007. 627 pp. ISBN: $852042564 X, 9788520425640$.

10. Tamplin J, Berlowitz DJ. A systematic review and meta-analysis of the effects of respiratory muscle training on pulmonary function in tetraplegia. Spinal Cord. 2014;52:175-80.

11. Kirshblum SC, Burns SP, Biering-Sorensen F, Donovan W, Graves DE, Jha A, et al. International standards for neurological classification of spinal cord injury (revised 2011). J Spinal Cord Med. 2011:34:535-46.
12. Barbas CV, Isola AM, Farias AM, Cavalcanti AB, Gama AM, Duarte A, et al. Brazilian recommendations of mechanical ventilation 2013. Part 2. Rev Bras Ter Intensiva. 2014;26:215-39.

13. Deford JA. Electroventilation. Am J Emerg Med. 1985;3:337-9.

14. Health Care Secretariat. Department of Strategic Programmatic Actions. Care guidelines for the person with spinal cord injury. vol. 1, 2nd ed. Brasilia: Ministry of Health; 2015. 68 pp. ISBN: 978-85-334-2229-2.

15. Bellucci $\mathrm{CH}$, de Castro Filho JE, Gomes CM, de Bessa J Jr, Battistella LR, De Souza DR, et al. Contemporary trends in the epidemiology of traumatic spinal cord injury: changes in age and etiology. Neuroepidemiology. 2015;44:85-90.

16. World Health Organization, International Spinal Cord Society. International perspectives on spinal cord injury. Switzerland: World Health Organization; 2013.

17. Chen Y, He Y, DeVivo MJ. Changing demographics and injury profile of new traumatic spinal cord injuries in the United States, 1972-2014. Arch Phys Med Rehabil 2016;97:1610-9.

18. Stein DM, Menaker J, McQuillan K, Handley C, Aarabi B, Scalea TM. Risk factors for organ dysfunction and failure in patients with acute traumatic cervical spinal cord injury. Neurocritical Care. 2010;13:29-39.

19. Goligher EC, Dres M, Fan E, Rubenfeld GD, Scales DC, Herridge MS, et al. Mechanical ventilation-induced diaphragm atrophy strongly impacts clinical outcomes. Am J Respir Crit Care Med. 2018;197:204-13.

20. Postma K, Haisma JA, Hopman MT, Bergen MP, Stam HJ, Bussmann JB. Resistive inspiratory muscle training in people with spinal cord injury during inpatient rehabilitation: a randomized controlled trial. Phys Ther. 2014;94:1709-19.

21. Pizano CA, Tarossi M, Tonella RM, Rodrigues CDA, Lima NMFV, Mandu S, et al. The effectiveness of the progressive orthostatism training in the pulmonary re-expansion in high spinal cord injury. Rev Brasileira Fisiol Exercício. 2013;12:40-4.

22. Vorona S, Sabatini U, Al-Maqbali S, Bertoni M, Dres M, Bissett $\mathrm{B}$, et al. Inspiratory muscle rehabilitation in critically ill adults. A Syst Rev Meta-Anal Ann Am Thorac Soc 2018;15:735-44.

23. Bissett B, Gosselink R, Van Haren FM. Respiratory muscle rehabilitation in patients with prolonged mechanical ventilation: a targeted approach. Annual Update in Intensive Care and Emergency Medicine. 2020. Cham: Springer; 2020. p. 595-609.

24. Condessa RL, Brauner JS, Saul AL, Baptista M, Silva AC, Vieira SR. Inspiratory muscle training did not accelerate weaning from mechanical ventilation but did improve tidal volume and maximal respiratory pressures: a randomised trial. J Physiother. 2013;59:101-7. 